\title{
ANALYTICAL APPROACH TO DERIVE THE FINE PARTICLE DISPERSION PROPERTIES INHERENT IN NUMERICAL PARTICLE TRAJECTORY MODELS
}

\author{
Ching-Ho Lin and Len-Fu W. Chang \\ Graduate Institute of Environmental Engineering, National Taiwan University, Taipei, Taiwan
}

(First received 1 August 1995; in final form 4 January 1996)

\begin{abstract}
To validate a particle trajectory model as a first check, one usually applies the model in simulating the dispersion of fine particles in homogeneous and stationary turbulence and compared the simulation results with the classical result of Taylor's theory. In this paper we present an analytic procedure to derive the dispersion properties of fine particles in homogeneous and stationary turbulence according to the mechanisms of particle trajectory models. Our approach avoids the use of numerical integration and the finite number of Monte-Carlo trials. This can shed light on the model properties without the mask of errors originated from numerical integration and statistical trials. Based on our approach, nine particle trajectory models in the literature are examined. In addition, the effects of the time interval of numerical integration taken in the models are analyzed and discussed. Copyright (C) 1996 Elsevier Science Ltd.
\end{abstract}

\section{INTRODUCTION}

The problem of dispersion and/or deposition of particles in turbulent flows is important in many fields of engineering. The transport and dispersion of aerosols in air and water, the mixing of fuel droplets in combustion chambers, the dust flow in electrostatic precipitator and the wall losses of a turbulent sampling tube, etc. are typical problems.

Numerical models appearing in the literature for particle motion in turbulent flows are constructed on either an Eulerian approach or a Lagrangian approach. Eulerian approach treats both the fluid and particles as a continuous medium with different phases. The dispersion of particles is predicted by solving the transport equations of these two phases. The difficulty of Eulerian approach is to determine the eddy diffusivity coefficient of the particles. Furthermore, Eulerian approach encounters the more difficult problem while particles influenced by external force, such as gravitational force.

Models employing the Lagrangian method are usually known as trajectory models. In general, to simulate or predict the dispersion and/or deposition of particles in turbulent flow by the so-called Lagrangian trajectory models includes the following key features:

(1) the realization of fluid instantaneous fluctuating velocity by known turbulence statistics, e.g. mean quantities and the second moment quantities,

(2) an ad hoc relationship between the Lagrangian and Eulerian statistics needs to be established, such that the driving fluid velocity history for a particle at consecutive positions can be constructed,

(3) a numerical integration of the equations of particle motion is performed,

(4) the dispersion of a cluster of particles can be evaluated only by a large number of trials (the so-called Monte-Carlo method) by repeating steps (1)-(3).

This approach is first presented by Yuu et al. (1978), and the differences among the models are the generating methods of the instantaneous fluid velocity (e.g. Step (2)) and choice of time interval $\Delta t$ of numerical integration (Gosman and Ioannides, 1981; Shuen et al., 1983; Ormancey and Martinon, 1984; Kallio and Reeks, 1989; Zhou and Leschziner, 1991; Abuzeid et al., 1991; Berlemont et al., 1990; Lu et al., 1992, 1993a, b). Based on this approach, particle trajectory models had been used to predict or simulate the performance of a shrouded probe sampling in turbulent flow (Gong et al., 1993), wall deposition of 
aerosol particles in turbulent flow (Kallio and Reeks, 1989; Abuzeid et al., 1991), solid particle motion in grid-generated turbulent flows (Ormancey and Martinon, 1984; Zhou and Leschziner, 1991; Berlemont et al., 1990; Lu et al., 1993b), particle dispersion in turbulent round jets (Yuu et al., 1978; Shuen et al., 1983), and the mixing of fuel droplets in combustion chambers (Gosman and Ioannides, 1981).

The validity and suitability of a particle trajectory model can be checked only through statistical sense by comparing the simulation and experimental data. To validate their models as a first check, the developers of the models usually applied their model in predicting the dispersion of fine particle in homogeneous and stationary turbulence, and compared the prediction with the classical result of Taylor's theory (see Gosman and loannides, 1981; Shuen et al., 1983; Ormancey and Martinon, 1984; Kallio and Reeks, 1989; Berlemont et al., 1990; Zhou and Leschziner, 1991). Adopting this checking procedure is based on a simple belief that any model suitable for complicated flows must be able to retrieve the simplest asymptotic condition, i.e. the dispersion of fine particles in homogeneous and stationary turbulence. In addition, the fine particle case can be considered as the case of particles with relaxation time far smaller than the turbulent eddy time scale.

In this paper, we present an analytic procedure to derive the dispersion properties of fine particles in homogeneous and stationary turbulence according to the mechanisms of particle trajectory models. Our approach avoids the effects of using numerical integration and the bias due to the finite number of Monte-Carlo trials. The results may shed light on the model properties without the mask of errors originated from numerical integration and statistical trials during the aforementioned preliminary model checking. Based on our approach, nine particle trajectory models in the literature are examined. They are the models proposed by Yuu et al. (1978, donated YYHJ model), Gosman and Ioannides (1981, GI model), Shuen et al. (1983, SCF model), Kallio and Reeks (1989, KR model), Zhou and Leschziner (1991, ZL model), Abuzeid et al. (1991, ABA model), Ormancey and Martinon (1984, OM model), Berlemont et al. (1990, BDG model), and Lu et al. (1992, 1993a, b, LFA model). In addition, the effects of the time interval of numerical integration taken in these models are analyzed and discussed. A short review of these models is presented in the next section.

It should be noticed that only the models with the aforementioned key features can be analyzed by our approach. For example, our approach cannot extend to examine the model of Ahmadi and his co-workers ( $\mathrm{Li}$ and Ahmadi, 1992; Li et al., 1994), which has been successfully applied to aerosol particle dispersion and deposition in turbulent channel flows. In their model, the fluid fluctuating velocity is generated by a continuous Gaussian random field proposed by Kraichnan (1970).

\section{SUMMARY OF PARTICLE TRAJECTORY MODELS}

Particle trajectory models are developed to predict the motion of particles in complicated turbulent flow. In general, the complexity is due to the inhomogeneity and multi-dimensional nature of the turbulent flow. In addition, the equations of particle motion associated with the models may include the terms of particle inertia, gravitational force, drag force, lifting force, etc. In the following, the equations of particle motion are not presented, and only the one-dimensional mechanism of the models in homogeneous and stationary turbulence is described.

\subsection{YYHJ model}

In the YYHJ model (Yuu et al., 1978), the fluid Lagrangian integral time scale is used as the time interval of numerical integration (hereafter it will be called as the time interval), i.e.

$$
\Delta t_{i}=\mathfrak{I}_{\mathrm{L}}
$$

in which $\Delta t_{i}$ is the $i$ th time interval of particle traveling and $\Im_{\mathbf{L}}$ is the fluid Lagrangian integral time scale. The fluid fluctuating velocity which drives a specific particle on its 
traveling path is determined by

$$
u_{p}\left(t_{i}\right)=u^{\prime} G\left(t_{i}\right)
$$

in which $u_{p}\left(t_{i}\right)$ is the driving fluid fluctuating velocity, the subscript $p$ denotes on the particle path, $u^{\prime}$ is the root-mean-square of the fluid fluctuating velocity, and $G\left(t_{i}\right)$ is a random number drawn from a Gaussian probability density distribution of zero mean and unit standard deviation.

The fluid fluctuating velocities in two successive time intervals of particle traveling are assumed to be uncorrelated. Yuu et al. (1978) explained that each movement of a specific particle is controlled by a turbulent eddy with a specific Lagrangian integral time scale. As a result, when the particle travels after a time interval $\mathfrak{I}_{L}$, the particle can be considered as falling into another eddy that is independent of the previous eddy in its characteristics.

\subsection{GI and SCF models}

In the GI and SCF models (Gosman and Ioannides, 1981; Shuen et al., 1983), the time interval of particle traveling is determined with the minimum between the eddy lifetime $t_{\mathrm{e}}$ and the transit time $t_{\mathrm{t}}$,

$$
\Delta t_{i}=\min \left(t_{\mathrm{e}}, t_{\mathrm{t}}\right)
$$

The eddy lifetime $t_{\mathrm{e}}$ is equal to $\mathfrak{I}_{\mathrm{L}}$ and the transit time $t_{\mathrm{t}}$ is the time for a particle to pass through an eddy. A minor difference between the GI and SCF models is the method to estimate the eddy lifetime $t_{\mathrm{e}}$. Furthermore, the driving fluid fluctuating velocity in both models is determined by

$$
u_{p}\left(t_{i}\right)=(2 k / 3)^{1 / 2} G\left(t_{i}\right)
$$

in which $k$ is the local value of turbulent kinetic energy.

\subsection{KR model}

In the KR model (Kallio and Reeks, 1989), a random time scale, called an instantaneous eddy lifetime, serves as the time interval of particle traveling, i.e.

$$
\Delta t_{i}=E\left(t_{i}\right) \mathfrak{I}_{\mathrm{L}}
$$

in which $E\left(t_{i}\right)$ is a random number drawn from an exponential probability density distribution, i.e.

$$
f(x)=\mathrm{e}^{-x} \text { for } 0 \leqslant x \leqslant \infty
$$

in which $f(x)$ is the exponential probability density function with unit mean and defined in the range from zero to infinity. The method to generate the driving fluid fluctuating velocity in this model is same as in the YYHJ model, as shown in equation (2).

\subsection{ZL model}

Zhou and Leschziner (1991) assumed that the fluid driving velocity at successive time intervals are related by a time-correlation coefficient,

$$
u_{p}\left(t_{i}\right)=a u_{p}\left(t_{i-1}\right)+u^{\prime} \sqrt{1-a^{2}} G\left(t_{i}\right)
$$

and

$$
a=R_{p \mathrm{~L}}\left(\Delta t_{i}\right)=\overline{u_{p}\left(t_{i}\right) u_{p}\left(t_{i-1}\right)} / u^{\prime 2}
$$

in which $R_{p \mathrm{~L}}\left(\Delta t_{i}\right)$ is a time-correlation coefficient. The exponential-type of correlation function is used in this model, i.e.

$$
R_{p \mathrm{~L}}\left(\Delta t_{i}\right)=\exp \left(-\Delta t_{i} / \mathfrak{I}_{\mathrm{L}}\right)
$$




\subsection{ABA model}

Abuzeid et al. (1991) used a similar time-correlation concept as in the ZL model, but the form of the generation of the driving fluid fluctuating velocity is different. In the ABA model, the driving fluid fluctuating velocity is generated by

$$
u_{p}\left(t_{i}\right)=u^{\prime} \sqrt{\alpha} G\left(t_{i}\right)+u^{\prime} \sqrt{1-x} G\left(t_{i-1}\right)
$$

in which $\alpha$ is an empirical parameter, and $\alpha=0.2$ is suggested by Abuzeid et al. (1991). According to equation (10) and the definition in equation (8), a relationship between $x$ and $R_{p \mathrm{~L}}\left(\Delta t_{i}\right)$ can be deduced as

$$
R_{p \mathrm{~L}}\left(\Delta t_{i}\right)=\sqrt{\alpha(1-\alpha)} .
$$

\subsection{OM model}

The traveling paths of a fluid parcel and a specific particle, even if they start at the same location and at the same time, are different. This distinction is absent from the $\mathrm{ZL}$ and ABA models; however, it is included in the OM model. In OM model, trajectories of a fluid parcel and a specific particle, as they start at the same position and at the same time, are traced. The time interval in this model is limited to the range from zero to $\mathfrak{\Im}_{\mathrm{L}}$, that is

$$
\Delta t_{i}=\beta \mathfrak{\Im}_{\mathrm{L}} \text { and } \quad 0<\beta \leqslant 1 .
$$

The fluid fluctuating velocity on the fluid parcel path is generated by

$$
u_{f}\left(t_{i}\right)=u_{f}\left(t_{i-1}\right) F_{1}\left(t_{i}\right)+u^{\prime} F_{2}\left(t_{i}\right) G\left(t_{i}\right)
$$

where

$$
\begin{array}{lll}
F_{1}\left(t_{i}\right)=1, & F_{2}\left(t_{i}\right)=0 & \text { for } \beta<N\left(t_{i}\right) \leqslant 1, \\
F_{1}\left(t_{i}\right)=0, \quad F_{2}\left(t_{i}\right)=1 & \text { for } 0<N\left(t_{i}\right) \leqslant \beta
\end{array}
$$

in which the subscript $f$ denotes on a fluid parcel path, while $N\left(t_{i}\right)$ is a random number sampled from a uniform probability density distribution defined in the range from zero to unity. The random number $\mathfrak{I}_{\mathrm{L}} N\left(t_{i}\right)$ can be considered as a random eddy lifetime. If the random eddy lifetime is smaller than the time interval $\beta \mathfrak{I}_{\mathrm{L}}$, it denotes the fact that the eddy in which the particle just stayed has vanished; hence a new value of fluid fluctuating velocity is generated by equations (13) and (15). In the opposite case, the fluid fluctuating velocity in the previous time step is retained by equations (13) and (14). The fluid fluctuating velocity on the path of the specific particle is connected to the fluctuating velocity on the path of the fluid parcel with a spatial correlation function.

\section{7. $B D G$ model}

Berlemont et al. (1990) followed the same approach as Ormancey and Martinon (1984), but they provided a more general correlation function of the fluid fluctuating velocity. The fluid fluctuating velocity on the fluid parcel path is generated by

$$
u_{f}\left(t_{i}\right)=u^{\prime} \sum_{j=1}^{n} b_{i j} G\left(t_{j}\right)
$$

in which $n$ is the total number of time intervals of particle traveling. From equation (16), it can be shown that (Berlemont et al., 1990)

$$
\overline{u_{f}\left(t_{i}\right) u_{f}\left(t_{j}\right)}=u^{\prime 2} \sum_{k=1}^{n} b_{i k} b_{j k} .
$$


If the correlation coefficient $a_{i j}$ is defined as

$$
a_{i j}=\overline{u_{f}\left(t_{i}\right) u_{f}\left(t_{j}\right)} / u^{\prime 2}=R_{\mathbf{L}}(|j-i| \Delta t)
$$

in which $R_{\mathrm{L}}(|j-i| \Delta t)$ is the fluid Lagrangian autocorrelation coefficient, equations (17) and (18) render the relationship

$$
a_{i j}=\sum_{k=1}^{n} b_{i k} b_{j k} .
$$

Berlemont et al. (1990) computed $a_{i j}$ from the Frenkiel family of correlation functions, i.e.

$$
a_{i j}=\exp \left[-|j-i| \Delta t /\left(m^{2}+1\right) \mathfrak{I}_{\mathrm{L}}\right] \cos \left[m|j-i| \Delta t /\left(m^{2}+1\right) \mathfrak{I}_{\mathrm{L}}\right] .
$$

A method to calculate $b_{i j}$ was suggested by Berlemont et al. (1990). The fluid fluctuating velocity at the location of the specific particle is connected to the fluctuating velocity at the location of the fluid parcel just same as in the OM model.

\subsection{LFA model}

The LFA model (Lu et al., 1992, 1993a, b) adopted a same idea as the OM and BDG models. However, this model adopted an explicit form of the relationships in which the timewise and spacewise correlation functions are used to connect the driving velocity for particle and fluid parcel at consecutive points on their pathlines. These are

$$
u_{p}\left(t_{i}\right)=a c u_{p}\left(t_{i-1}\right)+u^{\prime} \sqrt{1-a^{2} c^{2}} G\left(t_{i}\right)
$$

and

$$
\begin{gathered}
a=R_{\mathrm{L}}\left(\Delta t_{i}\right)=\overline{u_{f}\left(t_{i}\right) u_{f}\left(t_{i-1}\right)} / u^{\prime 2}, \\
c=R_{\mathrm{E}}(\Delta s)=\overline{u_{f}\left(t_{i}\right) u_{p}\left(t_{i}\right)} / u^{\prime 2}
\end{gathered}
$$

in which $R_{\mathrm{E}}(\Delta s)$ is the fluid Eulerian autocorrelation coefficient. Lu et al. $(1992,1993 \mathrm{a}$, b) got the time correlation $a$ from Frenkiel family of correlation functions with $m=0$ (see equation (20)) and the spatial correlation coefficient $c$ from a correlation function of the same type but with $m=1$.

\section{DISPERSION IN HOMOGENEOUS TURBULENT FLOW}

In this section, the dispersion properties of fine particles in homogeneous and stationary turbulence will be deduced based on the mechanisms proposed by different particle trajectory models summarized in last section.

\subsection{Basic assumption}

When considering the dispersion of fine particles in homogeneous and stationary turbulent flow, the mean flow direction is assumed to be in the $x_{1}$-direction. The dispersion of fine particles is only due to the lateral fluctuating velocity along the $x_{2}$-direction. In addition, all particles are released at the origin $O(0,0)$ and their initial velocities in the $x_{2}$-direction are random values sampled from a Gaussian probability density distribution with zero mean and variance equal to the root-mean-square of the fluid fluctuating velocities.

\subsection{Analytic method}

By assuming the dispersed particles with very small relaxation time, the motion of the fine particles will exactly follow the turbulent flow. So, the velocity of a specific particle can be obtained by setting it equal to the fluid velocity on the traveling path of the particle, and not 
necessarily through integrating the equations of particle motion. Consequently, the displacement of a specific particle in the $x_{2}$-direction (throughout this paper, displacement, dispersion and velocity, are indicated to be in the $x_{2}$-direction) in the $i$ th time interval is expressed as

$$
\Delta y\left(t_{i}\right)=u_{p}\left(t_{i}\right) \Delta t_{i}
$$

in which $\Delta y\left(t_{i}\right)$ is the displacement of the particle in the $i$ th time interval $\Delta t_{i}$ and $u_{p}\left(t_{i}\right)$ is the velocity of the particle or the fluid fluctuating velocity in the $i$ th time interval. After $n$ time intervals, the total displacement of the particle is

$$
y\left(t_{i}\right)=\sum_{i=1}^{n} \Delta y\left(t_{i}\right)=\sum_{i=1}^{n} u_{p}\left(t_{i}\right) \Delta t_{i}
$$

The mean squared displacement of all particles is expressed as

$$
\overline{y^{2}\left(t_{n}\right)}=\overline{\left[\sum_{i=1}^{n} u_{p}\left(t_{i}\right) \Delta t_{i}\right]^{2}} \text {. }
$$

Clearly, the dispersion of fine particles in homogeneous and stationary turbulence will depend on the mechanisms for the realization of the fluid fluctuating velocity and the time interval adopted by different models. A theoretical prediction of the dispersion of fluid particles in homogeneous and stationary turbulence has already been proposed by Taylor (see Hinze, 1975):

and

$$
\overline{y^{2}(t)}=u^{2} t^{2} \quad \text { for } t \ll I_{L}
$$

$$
\overline{y^{2}(t)}=2 u^{\prime 2} \Im_{1} t \quad \text { for } t \gg \Im_{1} .
$$

This theoretical results can be used as checking standard to infer the suitability of different trajectory models.

\subsection{The dispersion properties of the particle trajectory models}

3.3.1. YYHJ, GI and SCF models. Under the assumptions in this work, the fluid fluctuating velocity and the time interval taken in the YYHJ, GI and SCF models use the same formula. Combining the time interval in equation (1) and the fluid fluctuating velocity in equation (2) into equation (26), it yields

$$
\overline{y^{2}\left(t_{n}\right)}=\overline{\left[\sum_{i=1}^{n} u^{\prime} G\left(t_{i}\right) \Im_{\mathrm{L}}\right]^{2} .}
$$

With the properties

$$
\overline{G\left(t_{i}\right) \overline{G\left(t_{j}\right)}}=0 \quad \text { for } i \neq j
$$

and

$$
\overline{G\left(t_{i}\right) G\left(t_{j}\right)}=1 \quad \text { for } i=j,
$$

equation (29) can be reduced to

$$
\overline{y^{2}\left(t_{n}\right)}=n u^{\prime 2} \Im_{\mathrm{L}}^{2}
$$

Using

$$
t_{n}=\sum_{i=1}^{n} \Delta t_{i}=\sum_{i=1}^{n} \boldsymbol{\Im}_{\mathrm{L}}=n \boldsymbol{\Im}_{\mathrm{L}}
$$

equation (32) is reduced to

$$
\overline{y^{2}\left(t_{n}\right)}=u^{\prime 2} \Im_{\mathrm{L}} t_{n} .
$$


Equation (34) is the dispersion of fine particles in homogeneous and stationary turbulence according to the YYHJ, GI and SCF models.

3.3.2. KR model. In this model, the time interval is expressed in equation (5) and the generating method of the fluid fluctuating velocity is the same as the YYHJ model, as shown in equation (2). Combining equations (5) and (2) into equation (26), it yields

$$
\overline{y^{2}\left(t_{n}\right)}=\overline{\left[\sum_{i=1}^{n} u^{\prime} G\left(t_{i}\right) E\left(t_{i}\right) \widetilde{J}_{L}\right]^{2}} \text {. }
$$

Using equations (30) and (31), and the fact that $E\left(t_{i}\right)$ and $G\left(t_{j}\right)$ are independent of each other, equation (35) is reduced to

$$
\overline{y^{2}\left(t_{n}\right)}=\left(u^{\prime} \mathfrak{I}_{\mathrm{L}}\right)^{2} \sum_{i=1}^{n} \overline{E^{2}\left(t_{i}\right)}
$$

With the properties

$$
\begin{gathered}
\overline{E\left(t_{i}\right)}=\int_{0}^{\infty} x \mathrm{e}^{-x} \mathrm{~d} x=1, \\
\overline{E^{2}\left(t_{i}\right)}=\int_{0}^{\infty} x^{2} \mathrm{e}^{-x} \mathrm{~d} x=2, \\
\overline{t_{n}}=\overline{\sum_{i=1}^{n} \Delta t_{i}}=\overline{\sum_{i=1}^{n} E\left(t_{i}\right) \mathfrak{I}_{\mathrm{L}}}=\mathfrak{I}_{\mathrm{L}} \sum_{i=1}^{n} \overline{E\left(t_{i}\right)}=n \mathfrak{I}_{\mathrm{L}}
\end{gathered}
$$

equation (36) is reduced to

$$
\overline{y^{2}\left(t_{n}\right)}=2 u^{\prime 2} \mathfrak{J}_{\mathrm{L}} \overline{t_{n}} .
$$

Equation (40) is the dispersion of fine particles in homogeneous and stationary turbulence according to the KR model.

3.3.3. $Z L$ and LFA models. For fine particle, the coefficient $c$ in equation (23) reduces to unity. Comparing the generating method of fluid fluctuating velocity in equations (21) and (7), we know that the two equations in the LFA and ZL models, respectively, have the same form. The fluid fluctuating velocity in equation (7) is transformed to the following form:

$$
u_{p}\left(t_{i}\right)=u^{\prime} a^{i} G\left(t_{0}\right)+u^{\prime} \sqrt{1-a^{2}} \sum_{j=1}^{i} a^{i-j} G\left(t_{j}\right)
$$

Since there is no specific limitation for the selection of time interval in these two models, a reasonable expression of the time interval is

$$
\Delta t_{i}=\beta \mathfrak{\Im}_{\mathrm{L}}
$$

in which $\beta$ is a real number greater than zero. Combining equations (41), (42) and (26) yields

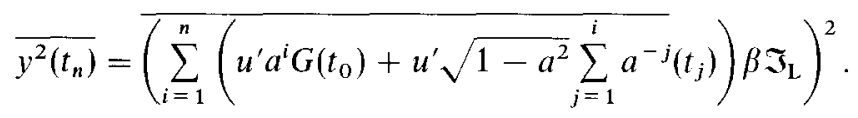

Furthermore, with the properties of equations (30) and (31), we obtain

$$
\overline{y^{2}\left(t_{n}\right)}=\left(\beta u^{\prime} \mathfrak{I}_{\mathrm{L}}\right)^{2}\left[\frac{a^{2}-2 a^{n+2}+a^{2 n+2}}{(1-a)^{2}}+\frac{\left(1-a^{2}\right)}{(1-a)^{2}}\left(n-\frac{2\left(a-a^{n+1}\right)}{(1-a)}+\frac{a^{2}-a^{2 n+2}}{\left(1-a^{2}\right)}\right)\right] .
$$

Using the property

$$
0<a=\mathrm{e}^{-\beta}<1
$$


and the condition for long dispersion time, i.e.

$$
n \beta \rightarrow \infty \text { and } n \rightarrow \infty,
$$

equation (44) is reduced to

$$
\overline{y^{2}\left(t_{n}\right)}=\left(\beta u^{\prime} \Im_{L}\right)^{2}\left[\frac{n(1+a)}{(1-a)}\right] .
$$

Using the relation,

$$
t_{n}=\sum_{i=1}^{n} \Delta t_{i}=\sum_{i=1}^{n} \beta \mathfrak{I}_{\mathrm{L}}=n \beta \mathfrak{I}_{\mathbf{L}}
$$

equation (47) can be expressed as

$$
\overline{y^{2}\left(t_{n}\right)}=\left[\beta \frac{(1+a)}{(1-a)}\right] u^{\prime 2} \Im_{1} t_{n}
$$

or

$$
\overline{y^{2}\left(t_{n}\right)}=\left[\beta \frac{\left(1+\mathrm{e}^{-\beta}\right)}{\left(1-\mathrm{e}^{-\beta}\right)}\right] u^{\prime 2} \mathbb{T}_{\mathrm{L}} t_{n} .
$$

Equation (50) is the dispersion of fine particles in homogeneous and stationary turbulence according to the ZL and LFA models at long dispersion time.

3.3.4. $A B A$ model. In this model, we express the time interval as equation (42). Substituting equations (42) and (10) into equation (26) yields

$$
\overline{y^{2}\left(t_{n}\right)}=\overline{\left[\sum_{i=1}^{n}\left(u^{\prime} \sqrt{x} G\left(t_{i}\right)+u^{\prime} \sqrt{1-\alpha} G\left(t_{i-1}\right)\right) \beta \mathfrak{J}_{\mathbf{L}}\right]^{2}} .
$$

By the properties of equations (30) and (31), equation (51) is further reduced to

$$
\overline{y^{2}\left(t_{n}\right)}=\left(\beta u^{\prime} \Im_{L}\right)^{2}[n+2(n-1) \sqrt{\alpha(1-\alpha)}] .
$$

Using equations (46) and (48), equation (50) can be expressed as

$$
\overline{y^{2}\left(t_{n}\right)}=[\beta(1+2 \sqrt{\alpha(1-\alpha)})] u^{\prime 2} \mathfrak{\Im}_{\mathrm{L}} t_{n} .
$$

Equation (53) is the dispersion of fine particles in homogeneous and stationary turbulence according to the ABA model at long dispersion time.

3.3.5. OM model. In this model, the time interval is expressed as in equation (12) and the fluid fluctuating velocity is expressed as in equations (13)-(15). The fluid velocity in equation (13) can be transformed to

$$
u_{p}\left(t_{1}\right)=u^{\prime}\left[F_{1}\left(t_{1}\right) G\left(t_{0}\right)+F_{2}\left(t_{1}\right) G\left(t_{1}\right)\right] \text { for } i=1
$$

and

$$
u_{p}\left(t_{i}\right)=u^{\prime}\left[G\left(t_{0}\right) \prod_{k=1}^{i} F_{1}\left(t_{k}\right)+\sum_{j=1}^{i-1}\left[G\left(t_{j}\right) F_{2}\left(t_{j}\right) \prod_{k=j+1}^{i} F_{1}\left(t_{k}\right)\right]+G\left(t_{i}\right) F_{2}\left(t_{i}\right)\right] \text { for } i \geqslant 2 .
$$

From the definitions in equations (13)-(15), the following properties can be obtained:

$$
\begin{gathered}
\overline{F_{1}^{2}\left(t_{i}\right)}=\overline{F_{1}\left(t_{i}\right)}=1-\beta, \\
\overline{F_{2}^{2}\left(t_{i}\right)}=\overline{F_{2}\left(t_{i}\right)}=\beta,
\end{gathered}
$$




$$
\begin{gathered}
\overline{F_{1}\left(t_{i}\right) F_{1}\left(t_{j}\right)}=\overline{F_{1}\left(t_{i}\right)} \overline{F_{1}\left(t_{j}\right)} \text { for } i \neq j, \\
\overline{F_{2}\left(t_{i}\right) F_{2}\left(t_{j}\right)}=\overline{F_{2}\left(t_{i}\right)} \overline{F_{2}\left(t_{j}\right)} \text { for } i \neq j, \\
\overline{F_{1}\left(t_{i}\right) F_{2}\left(t_{i}\right)}=0, \\
\overline{F_{1}\left(t_{i}\right) F_{2}\left(t_{j}\right)}=\overline{F_{1}\left(t_{i}\right)} \overline{F_{2}\left(t_{j}\right)} \text { for } i \neq j, \\
\overline{G\left(t_{i}\right) F_{1}\left(t_{j}\right)}=\overline{G\left(t_{i}\right)} \overline{F_{1}\left(t_{j}\right)}, \\
\overline{G\left(t_{i}\right) F_{2}\left(t_{j}\right)}=\overline{G\left(t_{i}\right)} \overline{F_{2}\left(t_{j}\right)} .
\end{gathered}
$$

Substituting the time interval in equation (12) and the velocity in equations (54), (55) into equation (26) yields

$$
\begin{aligned}
& \overline{y^{2}\left(t_{n}\right)}=\overline{\left(\left(u^{\prime} F_{1}\left(t_{1}\right) G\left(t_{0}\right)+u^{\prime} F_{2}\left(t_{1}\right) G\left(t_{1}\right)\right.\right.} \\
& +\overline{\left.\left.\sum_{i=2}^{n} u^{\prime}\left(G\left(t_{0}\right) \prod_{k=1}^{i} F_{1}\left(t_{k}\right)+\sum_{j=1}^{i-1}\left(G\left(t_{j}\right) F_{2}\left(t_{j}\right) \prod_{k=j+1}^{i} F_{1}\left(t_{k}\right)\right)+G\left(t_{i}\right) F_{2}\left(t_{i}\right)\right)\right) \beta \Im_{\mathrm{I}}\right)^{2}} .
\end{aligned}
$$

With the properties of equations (30) and (31) and the properties of equations (56)-(63), we finally obtain

$$
\overline{y^{2}\left(t_{n}\right)}=\left(\beta u^{\prime} \mathfrak{I}_{\mathrm{L}}\right)^{2}\left[\frac{n(2-\beta)}{\beta}+\frac{-2(1-\beta)+2(1-\beta)^{n+1}}{\beta^{2}}\right] .
$$

Using equations (46) and (48), equation (65) is reduced to

$$
\overline{y^{2}\left(t_{n}\right)}=[2-\beta] u^{\prime 2} \mathfrak{I}_{\mathrm{L}} t_{n} .
$$

Equation (66) is the dispersion of fine particles in homogeneous and stationary turbulence according to the $\mathrm{OM}$ model at long dispersion time.

3.3.6. BDG model. In this model, the time interval is expressed by equation (42). Substituting the fluid fluctuating velocity in equation (16) and the time interval in equation (42) into equation (26), it yields

$$
\overline{y^{2}\left(t_{n}\right)}=\overline{\left[\sum_{i=1}^{n}\left(u^{\prime} \sum_{j=1}^{n} b_{i j} G\left(t_{j}\right)\right) \beta \mathfrak{I}_{\mathrm{L}}\right]^{2}} .
$$

With the properties of equations (30) and (31), we obtain

$$
\overline{y^{2}\left(t_{n}\right)}=\left(\beta u^{\prime} \mathfrak{I}_{\mathrm{L}}\right)^{2} \sum_{i=1}^{n}\left[\sum_{j=1}^{n} b_{j i}\right]^{2} \text {. }
$$

With equation (19), equation (68) is reduced to

$$
\overline{y^{2}\left(t_{n}\right)}=\left(\beta u^{\prime} \mathfrak{I}_{\mathrm{L}}\right)^{2} \sum_{i=1}^{n} \sum_{j=1}^{n} a_{i j}
$$

Substituting the expression for $a_{i j}$ (equation (20)) into equation (69), and with some manipulations, we have

$$
\overline{y^{2}\left(t_{n}\right)}=\left(\beta \mathfrak{I}_{\mathrm{L}}\right)^{2}\left[n+\frac{2(n-1) \mathrm{e}^{-\beta}}{1-\mathrm{e}^{-\beta}}-\frac{2\left(\mathrm{e}^{-2 \beta}-\mathrm{e}^{-n \beta}\right)}{\left(1-\mathrm{e}^{-\beta}\right)^{2}}-\frac{2 \mathrm{e}^{-n \beta}}{1-\mathrm{e}^{-\beta}}\right]
$$


for $m=0$ in the correlation function of the Frenkiel family. And,

$$
\begin{aligned}
& \overline{y^{2}\left(t_{n}\right)}= \\
& \quad\left(\beta \Im_{\mathrm{L}}\right)^{2}\left[n+\operatorname{Re}\left\{\frac{2(n-1) \mathrm{e}^{-\left(1+i^{\prime}\right) \beta / 2}}{1-\mathrm{e}^{-\left(1+i^{\prime}\right) \beta / 2}}-\frac{2\left(\mathrm{e}^{-2\left(1+i^{\prime}\right) \beta / 2}-\mathrm{e}^{-n\left(1+i^{\prime}\right) \beta / 2}\right)}{\left(1-\mathrm{e}^{-\left(1+i^{\prime}\right) \beta / 2}\right)^{2}}-\frac{2 \mathrm{e}^{-n\left(1+i^{\prime}\right) \beta / 2}}{1-\mathrm{e}^{-\left(1+i^{\prime}\right) \beta / 2}}\right\}\right]
\end{aligned}
$$

in which $\operatorname{Re}\{\}$ is the real part in the brackets and $i^{\prime}=\sqrt{-1}$ for $m=1$ in the correlation function of the Frenkiel family. Using equations (46) and (48), equations (70) and (71), respectively, are reduced to

$$
\overline{y^{2}\left(t_{n}\right)}=\left[\beta \frac{1+\mathrm{e}^{-\beta}}{1-\mathrm{e}^{-\beta}}\right] u^{\prime 2} \Im_{\mathrm{L}} t_{n}
$$

and

$$
\overline{y^{2}\left(t_{n}\right)}=\left[\beta \frac{1-\mathrm{e}^{-\beta}}{1+\mathrm{e}^{-\beta}-2 \mathrm{e}^{-\beta / 2} \cos (\beta / 2)}\right] u^{\prime 2} \Im_{L} t_{n} .
$$

Equations (72) and (73) are the dispersion of fine particles at long dispersion time in homogeneous and stationary turbulence according to the BDG model for $m=0$ and $m=1$ in the correlation functions of the Frenkiel family, respectively.

\section{DISCUSSION}

In the following, we will focus on the comparison of the dispersion properties from model derivations and Taylor's theory for fine particles in homogeneous and stationary turbulence at long dispersion time (e.g. equation (28)). It should be noticed that the so-called long dispersion time as indicated by experiments (see Hinze, 1975) is valid when the traveling time of the fine particles is longer than several eddy time scales. In this situation, the dispersion behavior of the fine particles approaches to that of long dispersion time.

For the convenience of comparison, the dispersion of fine particles as derived from the models at long dispersion time can be expressed in a general form

$$
\overline{y^{2}\left(t_{n}\right)}=f(\beta) u^{\prime 2} \mathfrak{J}_{\mathrm{L}} t_{n}
$$

in which $f(\beta)$ is a "dispersion coefficient". Apparently, if the dispersion coefficient in a model depends on the value of $\beta$, it implies that the dispersion predicted by the model is influenced by the magnitude of the time interval taken in the model. Comparing equations (74) and (28), we know that a model to conform the Taylor's theory requires

$$
f(\beta)=2 .
$$

The dispersion coefficient $f(\beta)$ inherent in each models is summarized in Table 1. In Table 1, it is found that the values of $f(\beta)$ of the YYHJ, GI, SCF and KR models are constant. This is due to the fact that the time interval taken in these models has been limited in a specific way. For example, $\beta=1$ is used in the YYHJ, GI and SCF models. On the other hand, the $f(\beta)$ of the ZL, LFA, ABA, OM and BDG models is a function of $\beta$. It implies that the dispersions predicted from these models are dependant on the time interval taken in these models. In general, the users of a model may arrange the time interval as small as possible in simulating the particle motion in turbulent flows. So, the values of $f(\beta)$ at $\beta \leqslant 0.1$ for different models are calculated in Table 2. It shows that most models except ABA conform the Taylor's theory very well. This is true provided that the time intervals used in the models are kept small as compared to the eddy time scale. However, in some situations, it cannot avoid the choice of a large value of $\beta$. For example, when the model is applied to 
Table 1. Dispersion coefficient in particle trajectory models

\begin{tabular}{lll}
\hline Model & $\Delta t_{i}$ & $f(\beta)$ \\
\hline YYHJ, GI, SCF & $\widetilde{J}_{\mathrm{L}}$ & 1 \\
KR & $E\left(t_{i}\right) \cdot \mathfrak{J}$ & 2 \\
ZL, LFA & $\beta \widetilde{I}_{\mathrm{L}}$ & $\beta\left(1+\mathrm{e}^{-\beta}\right) /\left(1-\mathrm{e}^{-\beta}\right)$ \\
ABA & $\beta \mathfrak{I}_{\mathrm{L}}$ & $\beta(1+2 \sqrt{\alpha(1-\alpha)})$ \\
$\mathrm{OM}^{*}$ & $\beta \mathfrak{I}_{\mathrm{L}}$ & $2-\beta$ \\
$\mathrm{BDG}^{\dagger}(m=0)$ & $\beta \mathfrak{I}_{\mathrm{L}}$ & $\beta\left(1+\mathrm{e}^{-\beta}\right) /\left(1-\mathrm{e}^{-\beta}\right)$ \\
$\mathrm{BDG}^{\dagger}(m=1)$ & $\beta \mathfrak{I}_{\mathrm{L}}$ & $\beta\left(1-\mathrm{e}^{-\beta}\right) /\left(1+\mathrm{e}^{-\beta}-2 \mathrm{e}^{-\beta / 2} \cos (\beta / 2)\right)$ \\
\hline
\end{tabular}

* In the OM model, $\beta$ should be used in the range of $0<\beta \leqslant 1$.

${ }^{+}$The definition of $m$ is given in equation (20).

Table 2. Dispersion coefficient of particle trajectory models at $\beta \leqslant 0.1$

\begin{tabular}{llllll}
\hline & \multicolumn{5}{c}{$f(\beta)$ of models } \\
\cline { 2 - 5 }$\beta$ & ZL, LFA & ABA $^{*}$ & OM & $\begin{array}{l}\text { BDG }^{\dagger} \\
(m=0)\end{array}$ & $\begin{array}{l}\text { BDG }^{\dagger} \\
(m=1)\end{array}$ \\
\hline 0.01 & 2.0000 & 0.0180 & 1.9900 & 2.0000 & 2.0000 \\
0.02 & 2.0001 & 0.0360 & 1.9800 & 2.0001 & 2.0000 \\
0.03 & 2.0001 & 0.0540 & 1.9700 & 2.0001 & 2.0001 \\
0.04 & 2.0003 & 0.0720 & 1.9600 & 2.0003 & 2.0001 \\
0.05 & 2.0004 & 0.0900 & 1.9500 & 2.0004 & 2.0002 \\
0.06 & 2.0006 & 0.1080 & 1.9400 & 2.0006 & 2.0003 \\
0.07 & 2.0008 & 0.1260 & 1.9300 & 2.0008 & 2.0004 \\
0.08 & 2.0011 & 0.1440 & 1.9200 & 2.0011 & 2.0005 \\
0.09 & 2.0013 & 0.1620 & 1.9100 & 2.0013 & 2.0007 \\
0.10 & 2.0017 & 0.1800 & 1.9000 & 2.0017 & 2.0008 \\
\hline
\end{tabular}

* In ABA model, $\alpha=0.2$ is used (see equation (10)).

${ }^{+}$The definition of $m$ is given in equation (20).

particle motion near the solid wall, where the eddies are of very limited size and time scale. Figure 1 shows the variations of $f(\beta)$ when the values of $\beta$ are up to five. It shows that all the model predictions are deviating from Taylor's theory.

In Table 1, we find that the YYHJ, GI and SCF models which used a time interval determined by eddy integral time scale may underestimate the dispersion by a factor of one-half. This situation was also found by Kallio and Reeks (1989) in a numerical test. According to equations (29)-(34), if the time interval of these models is chosen as $2 \boldsymbol{I}_{\mathrm{L}}$ instead of $\mathfrak{\Im}_{\mathrm{L}}$, the dispersions predicted by these models may conform to Taylor's theory exactly. Furthermore, it is shown that the KR model, by using an instantaneous eddy lifetime concept to modify the YYHJ model, has been proven to be very successful. In KR model, the instantaneous eddy lifetime is realized from an exponential probability density function, i.e. equation (6). However, according to our analysis, the type of the probability density function can be relaxed to the following conditions:

$$
\overline{E\left(t_{i}\right)}=1
$$

and

$$
\overline{E^{2}\left(t_{i}\right)}=2
$$

The dispersion properties of ABA model depends on the determination of both the time interval and the empirical parameter $\alpha$, as shown in Table 1. Applying Taylor's theory as a guide, the time interval and the empirical parameter $\alpha$ have the relationship

$$
\beta(1+2 \sqrt{\alpha(1-\alpha)})=2
$$




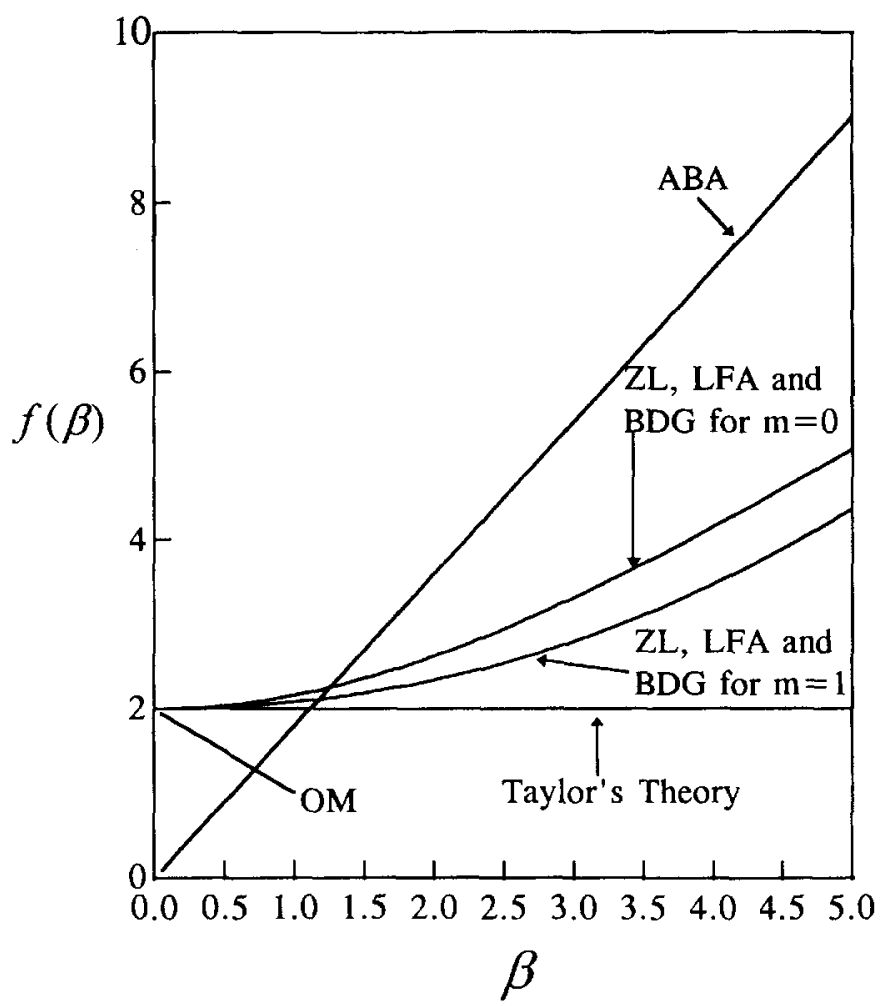

Fig. 1. The variations of $f(\beta)$ vs $\beta$ in particle trajectory models.

Abuzeid et al. (1991) suggested use of $\alpha=0.2$, this requires a value of

$$
\beta=1.1 \text {. }
$$

The relationship between $\alpha$ and the correlation function is expressed in equation (11). If the correlation function of exponential-type is used, the relationship between $\alpha$ and $\beta$ is

$$
\mathrm{e}^{-\beta}=\sqrt{\alpha(1-\alpha)}
$$

The solution of equations (78) and (80) yields

$$
\beta=1.29 \text { and } \alpha=0.92 \text { or } 0.08 \text {. }
$$

The results in equations (79) and (81) indicate that the time interval taken in the ABA model should be larger than the Lagrangian integral time scale.

For the OM model, as the time interval approaches zero, this model reveals the result of Taylor's theory, as shown in Fig. 1. By checking the driving fluid velocity in equations (13)-(15) in OM model, it can be seen that if $\beta=1$ is used in this model, the mechanism of this model will fall into the same form as YYHJ, GI and SCF models for fine particles in homogeneous and stationary turbulence. Also, this property is proved in our analysis as shown in Table 1 and Fig. 1 . They show that $f(\beta)=1$ at $\beta=1$ in OM model. This result is exactly the same as that of YYHJ, GI and SCF models.

In the ZL and LFA models, the correlation function of exponential-type (e.g. correlation function in the Frenkiel family with $m=0$ ) is used. Figure 1 reveals that, as the time interval approaches zero, the dispersion from these two models converges to the result of Taylor's theory. In the BDG model, the Frenkiel family of correlation functions with $m=0$ or $m=1$ can be used. For $m=0$, the BDG model shows the same dispersion results as the ZL and LFA models. In BDG model, the dispersions in both cases $m=0$ and $m=1$ approach Taylor's theory as $\beta$ approaches zero. However, the case with $m=1$ has a better prediction. 
If the correlation function with $m=1$ is used in the ZL and LFA model, we find the dispersion result are same as BDG models with $m=1$. In fact, if the Frenkiel family of correlation functions is used, a general form of dispersion coefficient in the ZL, LFA and BDG models can be expressed as

$$
f(\beta)=\beta \frac{1-\mathrm{e}^{-2 \beta /\left(m^{2}+1\right)}}{1+\mathrm{e}^{-2 \beta /\left(m^{2}+1\right)}-2 \mathrm{e}^{-\beta /\left(m^{2}+1\right)} \cos \left(m \beta /\left(m^{2}+1\right)\right)} .
$$

It should be noticed that the mechanisms to produce the driving fluid fluctuating velocity on the traveling path of a specific particle between the models of LFA (or ZL) and the BDG are quite different. The same dispersion results in these models as shown in equation (82) allows the users of models to apply the model with simpler mechanism.

\section{CONCLUSION}

An analytic approach is used in this paper to derive the dispersion properties of fine particles in homogeneous and stationary turbulence according to the mechanisms of different particle trajectory models. So, the analytic forms of dispersion associated with different models can be compared with the classical Taylor dispersion theory. This may provide us an understanding of model properties, the minor tune-up of the models with some empirical constants, and the determination of numerical time intervals associated with different models used to predict the dispersive motion of particles in turbulence. Some tentative conclusions can be drawn as follows:

(1) Most of particle trajectory models may conform to the Taylor's theory under the condition of a careful determination of some empirical constants associated with the time interval used in the model's numerical procedure.

(2) Particle trajectory models which adopt the eddy lifetime scale as the time interval of their particle dispersive motion in turbulence may improve their predictions as compared to Taylor's theory, if the time interval could be tuned up to twice the Lagrangian integral time scale. This includes the models of YYHJ, GI and SCF.

(3) The group of models which adopt a time-correlation coefficient to connect the turbulent fluctuating velocity at a consecutive time interval, or at consecutive positions may predict the Taylor's result exactly as long as the time interval in numerical procedure kept much smaller than eddy time scale. This group of models include OM, ZL, LFA and BDG models.

(4) When the dispersed particles are of larger relaxation time as compared with the local eddy time scale, we think the major mechanisms responsible for the total dispersion are more complicated than the situations that we are treating here. However, we still believe that the findings in this paper may provide a good rule of thumb to design a particle trajectory model applied to complicated dispersion process.

\section{REFERENCES}

Abuzeid, S., Busnaina, A. A. and Ahmadi, G. (1991) Wall deposition of aerosol particles in a turbulent channel flow. J. Aerosol Sci. 22, 43-62.

Berlemont, A., Desjonqueres, P. and Gouesbet, G. (1990) Particle Lagrangian simulation in turbulent flows. Int. J. Multiphase Flow 16, 19-34.

Gong, H., Anand, N. K. and McFarland, A. R. (1993) Numerical prediction of the performance of a shrouded probe sampling in turbulent flow. Aerosol Sci. Technol. 19, 294-304.

Gosman, A. D. and Ioannides, E. (1981) Aspects of computer simulation of liquid-fuelled combustors. AI AA paper 81-0323, 19th Aerospace Science Meeting, St. Louis.

Hinze, J. Q. (1975) Turbulence. McGraw-Hill, New York.

Kallio, G. A. and Reeks, M. W. (1989) A numerical simulation of particle deposition in turbulent boundary layers. Int. J. Multiphase Flow 15, 443-446.

Kraichnan, R. H. (1970) Diffusion by a random velocity field. Physics Fluids 13, 22-31.

Li, A. and Ahmadi, G. (1992) Dispersion and disposition of spherical particles from point sources in a turbulent channel flow. Aerosol Sci. Technol. 16, 209-226.

Li, A. and Ahmadi, G., Bayer, R. G. and Gaynes, M. A. (1994) Aerosol particle deposition in an obstructed turbulent duct flow. J. Aerosol Sci. 25, 91-112. 
Lu, Q. Q., Fontaine, J. R. and Aubertin, G. (1992) Particle motion in two-dimensional confined turbulent flows. Aerosol Sci. Technol. 17, 169-185.

Lu, Q. Q., Fontaine, J. R. and Aubertin, G. (1993a) Particle motion in shear turbulent flow. Aerosol Sci. Technol. 18, 85-99.

Lu, Q. Q., Fontaine, J. R. and Aubertin, G. (1993b) Numerical study of the solid particle motion in grid-generated turbulent flows. Int. J. Heat Mass Transfer 36, 79-87.

Ormancey, A. and Martinon, J. (1984) Prediction of particle dispersion in turbulent flow. Physico-chem. Hydrodynam. 5, 229-240.

Shuen, J.-S., Chen, L.-D. and Faeth, G. M. (1983) Evaluation of a stochastic model of particle dispersion in a turbulent round jet. AIChE J.29, 167-170.

Yuu, S., Yasukouchi, N., Hirosawa, Y. and Jotaki, T. (1978) Particle turbulent diffusion in a dust-laden round jet. AIChE J. 24, 509-519.

Zhou, Q. and Leschziner, M. A. (1991) A Lagrangian particle dispersion model based on a time-correlated stochastic approach. Gas-Solid Flows ASME FED-121, 255-260. 